\title{
A benchmark study for CHIANTI based on RESIK solar flare spectra
}

\author{
C. Chifor ${ }^{1}$, G. Del Zanna ${ }^{2}$, H. E. Mason ${ }^{1}$, J. Sylwester ${ }^{3}$, B. Sylwester ${ }^{3}$, and K. J. H. Phillips ${ }^{2}$ \\ 1 Department of Applied Mathematics and Theoretical Physics, Centre for Mathematical Sciences, Wilberforce Road, \\ Cambridge CB3 0WA, UK \\ e-mail: [c.chifor;h.e.mason]@damtp.cam.ac.uk \\ 2 Mullard Space Science Laboratory, University College London, Holmbury St. Mary, Dorking, Surrey RH5 6NT, UK \\ e-mail: [gdz;kjhp]@mssl.ucl.ac.uk \\ 3 Space Research Centre, Polish Academy of Sciences, 51-622, Kopernika 11, Wroclaw, Poland \\ e-mail: [js;bs]@cbk.pan.wroc.pl
}

Received 18 August 2006 / Accepted 12 October 2006

ABSTRACT

\begin{abstract}
Aims. To perform a benchmark analysis for the recent version of the CHIANTI atomic database (v. 5.2) based on high-resolution solar flare X-ray spectra in the range 3.4-6.1 $\AA$ from the RESIK crystal spectrometer on the CORONAS- $F$ spacecraft.

Methods. A C5.8 flare occurring on 2003 February 22 was chosen for analysis. RESIK spectra of this flare include emission lines of He-like and $\mathrm{H}$-like $\mathrm{K}, \mathrm{Ar}, \mathrm{S}$, and $\mathrm{Si}$, with some dielectronic lines. Initially, two independent plasma diagnostic techniques are employed: an emission measure $(E M)$ loci analysis using the line flux and the line contribution function $G\left(T_{\mathrm{e}}, N_{\mathrm{e}}\right)$, and a new method based on continuum fluxes and contribution functions. We further apply a differential emission measure (DEM) analysis, from which CHIANTI synthetic spectra are derived. The continuum from RESIK spectra is checked against simultaneous RHESSI and GOES observations. Comparisons of CHIANTI synthetic spectra with those from the MEKAL code in the 3.4-6.1 A range are also presented. Results. The emitting plasma appears multi-thermal, having one dominant temperature component determined independently from the line and continuum EM loci and DEM analyses. Consistency between line and continuum emissions requires photospheric elemental abundances (Asplund et al. 2005), with a depleted sulphur abundance. With the exception of RESIK channel 4 (5.0-6.1 Å), we find overall very good agreement between the calculated and observed intensities. From comparisons with other instruments, RESIK's precision in the continuum level is confirmed to be within the estimated $20 \%$ uncertainties in the intensity calibration. We find general agreement between CHIANTI and MEKAL isothermal spectra, but we note that the atomic data for the Si XII and Si XIII ions contained in CHIANTI are more complete.

Conclusions. RESIK observations of both lines and continua are suitable for characterising the properties of the flaring plasma such as temperature, emission measure and elemental abundance. These spectra can be used to evaluate any atomic database.
\end{abstract}

Key words. line: identification - plasmas - Sun: flares - Sun: X-rays, gamma rays

\section{Introduction}

Soft X-ray spectra from solar active regions and flares are useful in providing information about the emitting plasma properties such as temperature, density or elemental abundance. For our understanding of the physics behind the high-energy phenomena occurring in the solar atmosphere, it is crucial to know the values of these physical quantities. Analyses of spectral line and continuum emission enable measurements of the physical plasma parameters. Over the years, improved plasma diagnostics studies and line identification have been made possible through an enhanced knowledge of atomic physics in the X-ray range. Several different atomic databases and codes have been developed.

The CHIANTI package (Dere et al. 1997; Landi et al. 2006), increasingly used in the astrophysics community, contains upto-date atomic data which include atomic energy levels, theoretical and observed wavelengths and collision strengths as well as diagnostics tools for astrophysical plasmas. As for any atomic code, accuracy and completeness are of great importance. For this purpose, ongoing work has been carried out to benchmark the atomic data against observations. A benchmark study for the MEKAL (Mewe-Kaastra-Liedahl) atomic code was carried out by Phillips et al. (1999a). Previous benchmark analyses carried out for the CHIANTI atomic database in the soft X-rays include work by Landi \& Phillips (2005) in the wavelength range 7.47$18.97 \AA$, based on data from the Flat Crystal Spectrometer of the soft X-ray Polychromator (Acton et al. 1980) on board the Solar Maximum Mission (SMM). A series of benchmark studies, on an ion by ion basis, is also underway (Del Zanna et al. 2005, (Fe XXIII); Del Zanna 2006a, (Fe XXIV); Del Zanna 2006b, (Fe XVIII)).

The REntgenovsky Spektrometr s Izognutymi Kristalami (RESIK: Sylwester et al. 2005), one of the instruments on board the Russian CORONAS- $F$ mission, was in operation between July 2001 and May 2003. RESIK is a high-resolution crystal spectrometer observing in the range 3.4-6.1 $\AA$, a wavelength range which has been explored very little by previous spectrometers. Observed spectra include the diagnostically important lines of He-like and H-like Ar, K, S, and Si ions, as well as associated dielectronic satellites. Considerable work has been done on the intensity calibration of RESIK, with uncertainties estimated to be as small as $20 \%$ in the first diffraction order of the instrument. It is, therefore, timely to perform a benchmark of the most up-to-date atomic data using the latest calibrated RESIK spectra.

In the present work, we perform a benchmark analysis based on the atomic data contained in the CHIANTI database (version 5.2) and RESIK observations. At the same time, we use 
the CHIANTI package as a diagnostics toolbox to determine the physical properties of flaring plasma. Given the fact that MEKAL data have been widely used in the past, the question often arises as to whether any significant differences with the latest atomic data are present. A detailed comparison between the two sets of data (CHIANTI and MEKAL) and their application to X-ray stellar observations from Chandra was performed by Del Zanna (2002) using CHIANTI v. 4. In this paper, we use CHIANTI v. 5.2 data, comparing them with MEKAL predictions as well as the RESIK observations of solar flare X-rays.

An advantage that RESIK has over most previous crystal spectrometers is the fact that it observed flare continua with a negligible instrumental background in two of its 4 channels. This property, as well as the precision of the calibration (expected 20\% uncertainty), make RESIK suitable for comparisons and joint analysis with other solar instruments sensitive to soft X-rays. As shown in preliminary work by Sylwester et al. (2005) and Dennis et al. (2005), agreement between RESIK continua of initial spectra and RHESSI low energy spectra (above $4 \mathrm{keV}$ ) is satisfactory. Examples of such cross-calibrations between the recently calibrated RESIK spectra, RHESSI and GOES are also given in this paper.

In Sect. 2 we describe the RESIK flare observations used in our study, while cross-calibrations between RESIK, RHESSI and GOES are described in Sect. 3. The atomic codes benchmarked during our analysis are outlined in Sect. 4 . The plasma diagnostic techniques used to obtain the values of the physical parameters characterising the emitting plasma are described in Sect. 5. Finally, Sect. 6 includes a discussion and our conclusions.

\section{Observations of the 2003 February 22 flare}

We analysed RESIK spectra taken during a low intensity flare of GOES class C5.8 which occurred in the NOAA 10290 (16N 05W) disk active region. Since RESIK is an uncollimated spectrometer, spectral confusion may result when another active region is present on the Sun. In order to assess the solar activity, we investigated full disk images taken by the EIT (Extreme-ultraviolet Imaging Telescope: Delaboudinière et al. 1995) aboard the Solar and Heliospheric Observatory (SoHO) in the $195 \AA$ (Fe XII) filter (Fig. 1 top panel). The Transition Region and Coronal Explorer (TRACE: Handy et al. 1999) also observed the flaring region in the $195 \AA$ pass-band with higher spatial resolution. Figure 1 bottom panel shows the TRACE image taken during the flare peak at around 09:29 UT, with contours of X-ray emission from the Reuven Ramaty High Energy Solar Spectroscopic Imager (RHESSI: Lin et al. 2002). Although we notice other active regions present on the solar disk on 2003 Feb. 22, no separate significant flaring emission was detected during the time of the flare we chose to analyse.

An enhancement in soft X-ray emission started at 09:27 UT, with a flare maximum attained at 09:29 UT and the decay lasting until after 09:36 UT. The event was recorded by RESIK operating in first order Bragg-diffraction. An analysis of the plasma parameters is presented by Sylwester et al. (2006a). Figure 2 shows the temporal evolution of the flare in X-rays detected by RHESSI, RESIK and GOES. Both RHESSI and CORONAS-F spacecraft emerged from particle radiation belts just before the beginning of the flare. RHESSI observed the flare with the thin attenuator ("A1" state) in place.

RESIK spectra averaged over three stages during the flare rise, peak and decay (09:28-09:29 UT, 09:29-09:31 UT and 09:31-09:38 UT respectively) are displayed in Fig. 3, with the strongest emission lines observed indicated in the top panel.
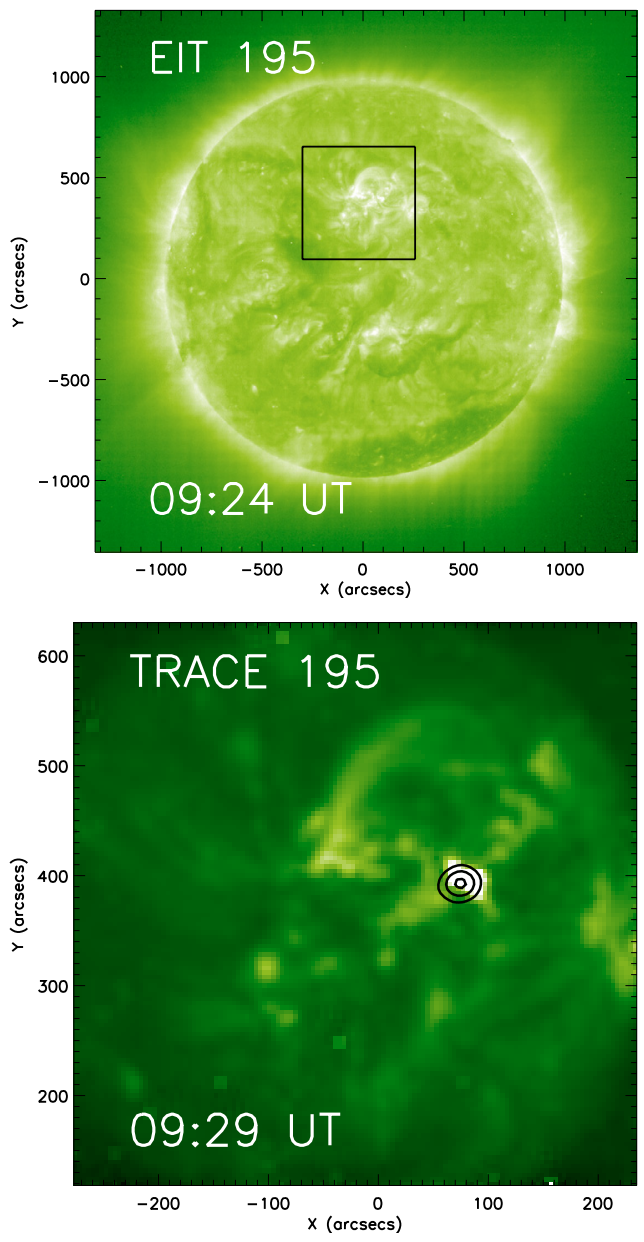

Fig. 1. Top panel: Full-disk image in the EIT $195 \AA$ A band taken shortly before the 2003 Feb. 22 flare event. The rectangular box marks the FOV of the TRACE image shown in the bottom panel. Bottom panel: TRACE $195 \AA$ image taken during the peak of the flare with contours drawn at $0.2,0.5$ and 0.9 of the maximum emission in the $3-12 \mathrm{keV}$ X-ray range as imaged by RHESSI between 09:28:50-09:29:10 UT.

To measure the RESIK line fluxes we used the $x c f i t$ routine available in the $\mathrm{SoHO}$ - CDS library, iteratively fitting Gaussian profiles to the observed lines. In practice, the Gaussian profiles are acceptable for small reduced values of $\chi^{2}$, although, strictly defined, the observed RESIK profiles are due to the thermal Doppler broadening, possible nonthermal broadening and a nonGaussian, instrumental profile. The RESIK continuum was measured by fitting polynomial functions to the observed spectra in regions where line emission is relatively small.

\section{Cross-calibrations between RESIK, RHESSI and GOES}

In order to increase the level of confidence in the calibration of RESIK spectra, it is possible to complement RESIK observations with simultaneous X-ray data from other solar instruments such as RHESSI and GOES. RHESSI was launched in February 2002 and continues to make imaging and spectroscopic observations of solar flares in both X-rays and gamma rays. Thermal free-free and free-bound continuum together with emission lines are seen in the X-ray range $\sim 5-20 \mathrm{keV}$ from flare plasmas with temperatures from $\sim 7 \mathrm{MK}$ to tens of MK (Phillips 2004). Comparisons between the lower end of RHESSI spectral range (background subtracted) and RESIK channels 1-4 (in 


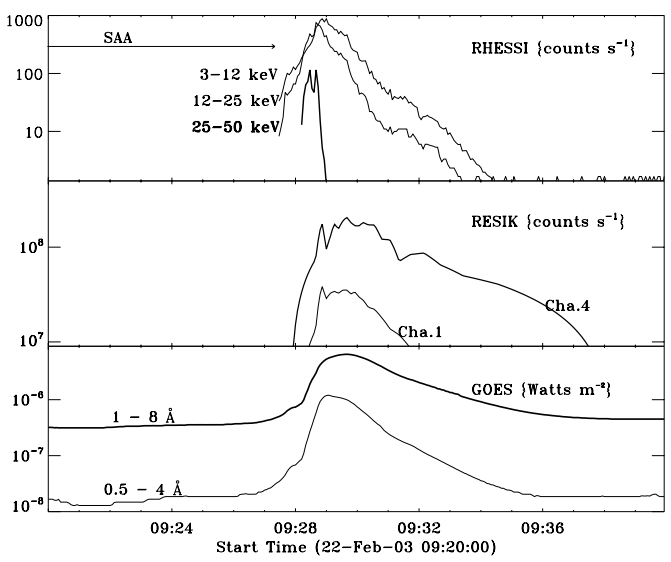

Fig. 2. Temporal evolution in X-rays during the 2003 Feb. 22 flare. Top panel: RHESSI light curves in the energy ranges of $3-12 \mathrm{keV}$, $12-25 \mathrm{keV}$, and 25-50 keV averaged over RHESSI detectors 1, 3, 4, $5,6,8$, and 9. During the flare, RHESSI's thin attenuator was placed in front of the detectors. Both RHESSI and RESIK emerge from the South Atlantic Anomaly (SAA) at about 09:27 UT. Middle panel: RESIK integrated counts in the ranges 3.4-3.8 $\AA$ (channel 1) and 5.0-6.1 (channel 4). Bottom panel: GOES fluxes in the 0.5-4 $\AA$ and 1-8 Å channels.

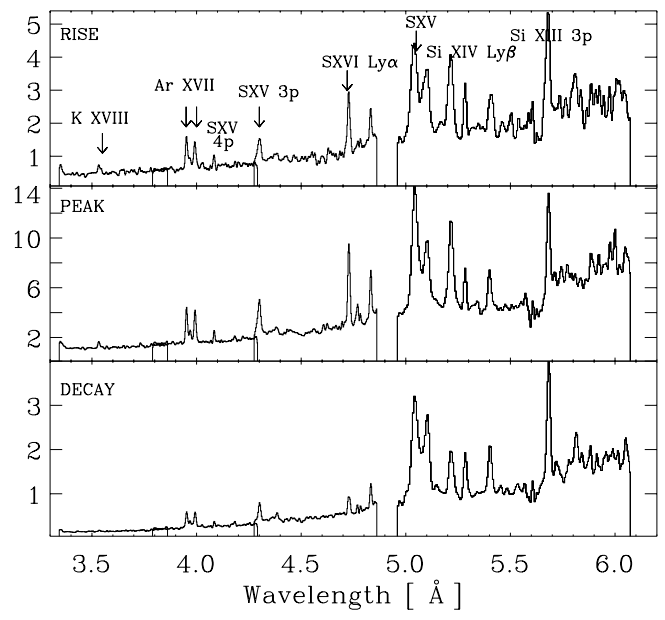

Fig. 3. RESIK averaged spectra during the flare rise (09:2809:29 UT), at flare peak (09:29-09:31 UT) and during flare decay (09:31-09:38 UT). Units are $10^{5}$ photons $\mathrm{cm}^{-2} \mathrm{~s}^{-1} \AA^{-1}$. Identifications of the most prominent lines are displayed in the top panel.

first order diffraction) were made at different times during the flare. Figure 4 shows one example of such comparison for spectra taken during the flare rise ( 09:29 UT).

GOES continua were obtained from the temperature and emission measure parameters derived from the ratio of the two GOES channels (0.5-4 $\AA$ and 1-8 $\AA$ ) using functions describing the thermal continuum. We used the recent software described by White et al. (2005), which allows for the possibility of including continuum functions from either MEKAL or CHIANTI v. 5.1 atomic models (both are shown in Fig. 4). Although the GOES routines are not updated to include the CHIANTI v. 5.2, we note that the continua calculated using either of the two versions (5.1 and 5.2) do not differ.

The calibration of RHESSI below $\sim 4 \mathrm{keV}$ in the $\mathrm{A} 1$ attenuator state has significant uncertainties (Smith et al. 2002), so we only plot the observed RHESSI spectrum above this energy. By extrapolating the RHESSI fitted spectrum we find close agreement between RESIK and RHESSI continua levels. Good

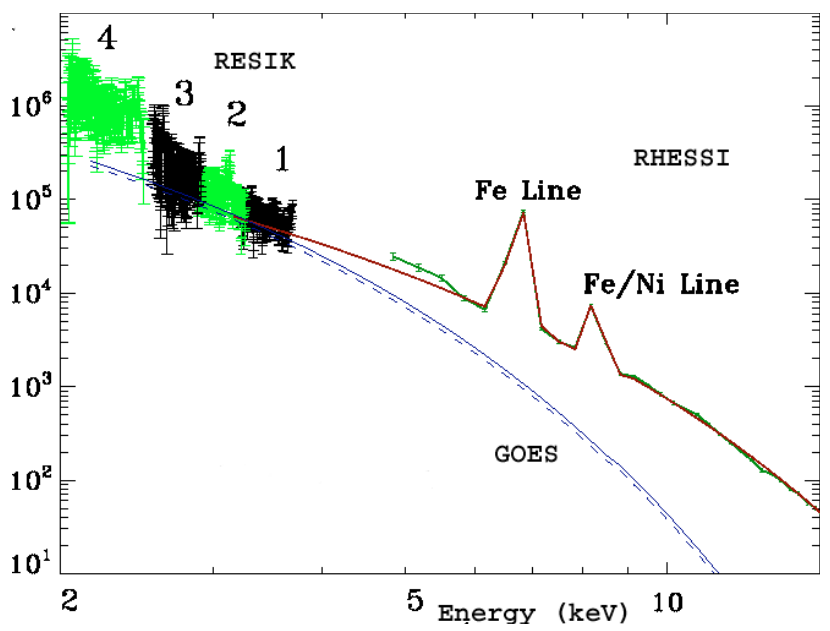

Fig. 4. Cross-calibration between RESIK, RHESSI and GOES during the 2003 Feb. 22 flare rise ( 09:29 UT). Spectra are plotted against energy in $\mathrm{keV}$ units, while fluxes are measured in photons $\mathrm{s}^{-1} \mathrm{~cm}^{-2} \mathrm{keV}^{-1}$. RESIK channels $1-4$ are plotted in different grey tones. Overplotted is a spectrum taken with RHESSI's detector 4, having the thin attenuator in place (A1 state). The modelled RHESSI spectrum is also shown, extrapolated at lower energies. The $\mathrm{Fe}$ and $\mathrm{Fe} / \mathrm{Ni}$ line features detected by RHESSI are clearly visible. GOES spectra are obtained from the temperature and emission measure parameters derived from the ratio of the two GOES channels using functions describing the thermal continuum based on the MEKAL atomic model (dashed), and the CHIANTI v. 5.1 model (solid).

agreement in temperatures, however, is achieved only during later decay stages of the flare. This may be attributed to a nonisothermal nature of flare plasma close to the flare peak and shortly after. Since RHESSI is sensitive to higher energy emission than RESIK, it is likely that it detected a higher temperature component during the flare impulsive phase. The $\mathrm{Fe}$ and $\mathrm{Fe} / \mathrm{Ni}$ features at $\sim 6.7 \mathrm{keV}$ and $\sim 8 \mathrm{keV}$ are present in RHESSI spectra, especially at the flare peak and shortly after. These features, due to many highly ionised $\mathrm{Fe}$ and $\mathrm{Ni}$ lines, are a good indicator of temperatures $\gtrsim 20 \mathrm{MK}$ when both are clearly visible in RHESSI spectra (Phillips et al. 2006b). We note that RESIK can also observe the $\mathrm{Fe}$ and $\mathrm{Fe} / \mathrm{Ni}$ complexes in its third diffraction order. However, at the time of the 2003 Feb. 22 flare, the instrument only operated in its first order mode.

\section{Comparison between the CHIANTI and MEKAL atomic data in the RESIK wavelength range}

The latest version (5.2) of the CHIANTI atomic database and programs was used for predicting line wavelengths and fluxes. We first compared predictions from CHIANTI with synthetic lines and continua obtained with the MEKAL code. A direct comparison between different atomic codes is not straightforward, with the number of lines and some corresponding wavelengths being different in each code. MEKAL predictions were obtained using the linflx and conflx routines available in the SolarSoft package. CHIANTI allows users to select density, emission measure, ionisation fractions and abundances in order to obtain simulated spectra. To achieve a meaningful comparison, we input into CHIANTI the default parameters for MEKAL. Solar coronal abundances from Meyer (1985) and ionisation fractions from Arnaud \& Rothenflug (1985) were used. Synthetic spectra were obtained for an electron density of $10^{8} \mathrm{~cm}^{-3}$ and a volume $E M$ of $10^{44} \mathrm{~cm}^{-3}$ with an isothermal 

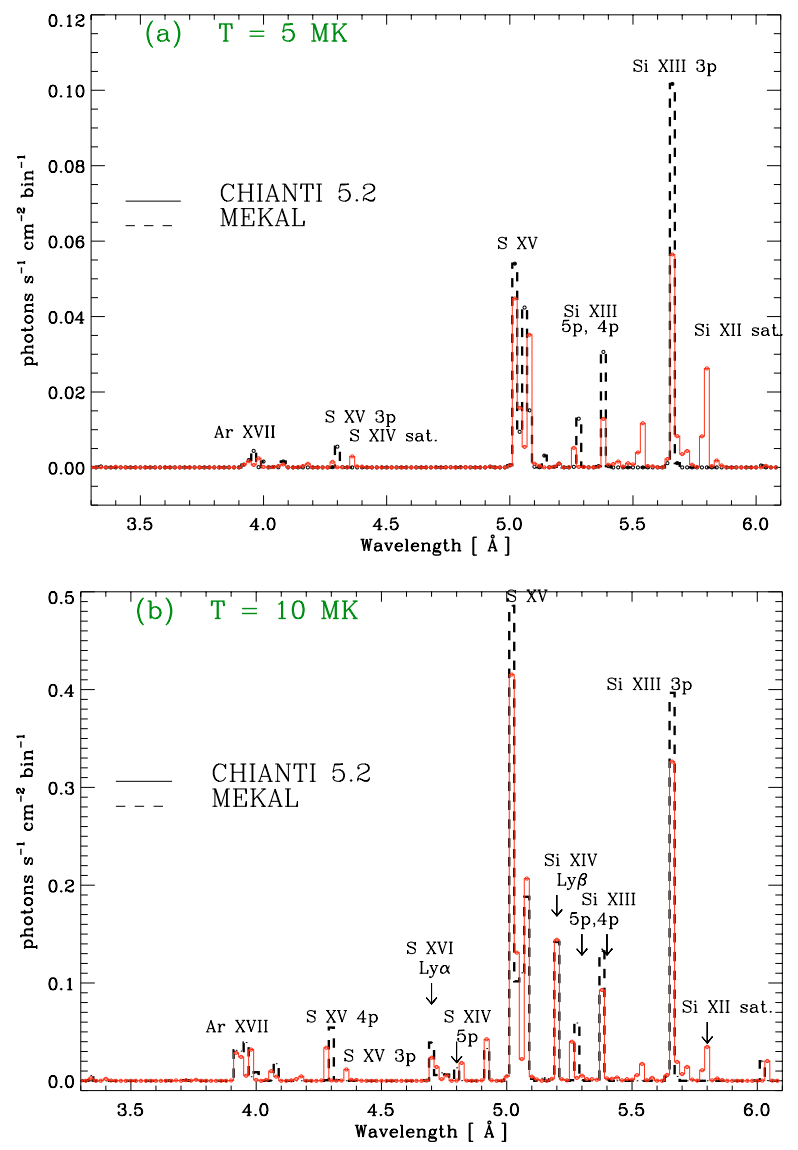

Fig. 5. Direct comparison of CHIANTI v. 5.2 (solid) and MEKAL (dashed) line intensities in the 3.4-6.1 $\AA$ range for an isothermal emitting plasma at $5 \mathrm{MK} \mathbf{a}$ ) and $10 \mathrm{MK} \mathbf{b})$. Ionisation fractions from Arnaud \& Rothenflug (1985) and Meyer (1985) coronal abundances used in MEKAL were also input into CHIANTI.

approximation at 5 and $10 \mathrm{MK}$ (Fig. 5). A steady state ionisation equilibrium was assumed.

Comparisons indicate good overall agreement between the two codes. We observe differences in wavelengths $(<10 \mathrm{~m} \AA)$ for the $S$ XV $\left(1 s^{2}-1 s 3 p\right)$ line at $4.30 \AA$ and the Si XIII $\left(1 s^{2}-1 s 5 p\right)$ line at $5.29 \AA$. Nevertheless, these differences are not significant at the RESIK resolution. At $5 \mathrm{MK}$, discrepancy was found between the predicted fluxes of the Si XIII $\left(1 \mathrm{~s}^{2}-1 \mathrm{~s} 3 \mathrm{p}\right)$ line at $5.68 \AA$, with a more intense line (by approximately $100 \%$ ) from the MEKAL data. CHIANTI also includes the Li-like Si (Si XII) dielectronic lines at 5.82 and $5.56 \AA$ which are not included in MEKAL. These lines have been studied in detail by Phillips et al. (2006a). Figure 6 shows the agreement between the thermal (free-free, free-bound and two-photon) CHIANTI and MEKAL continua at 5, 10 and $20 \mathrm{MK}$.

\section{Plasma diagnostics}

In order to obtain CHIANTI synthetic spectra for direct comparison with RESIK observations, we need to know the properties of the emitting plasma. The different methods we used to obtain the physical parameters of the flaring plasma are described below.

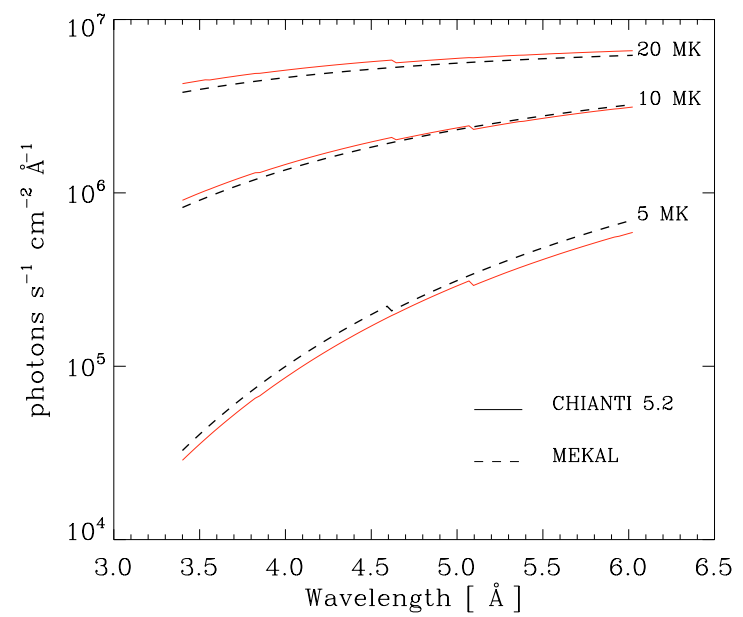

Fig. 6. Continuum obtained from CHIANTI v. 5.2 (solid line) compared with continuum obtained from MEKAL (dashed line). Ionisation fractions from Arnaud \& Rothenflug (1985) and Meyer (1985) coronal abundances were used for both MEKAL and CHIANTI.

\subsection{Line emission measure loci analysis}

As a first diagnostic method, we applied an EM loci analysis (see e.g. Jordan et al. 1987; Del Zanna et al. 2002) to RESIK spectroscopic lines. The flux observed at Earth (measured in photons $\mathrm{cm}^{-2} \mathrm{~s}^{-1}$ ) from an optically thin spectral line is given by

$F=\frac{1}{4 \pi(1 \mathrm{AU})^{2}} \int G\left(T_{\mathrm{e}}, N_{\mathrm{e}}\right) N_{\mathrm{e}}^{2} \mathrm{~d} V$

where $T_{\mathrm{e}}$ and $N_{\mathrm{e}}$ are the electron temperature and density, $G\left(T_{\mathrm{e}}, N_{\mathrm{e}}\right)$ is the line contribution function, $V$ is the volume of the emitting plasma and $1 \mathrm{AU}$ is the Earth-Sun distance. The contribution function, containing all relevant atomic physics parameters, peaks strongly with temperature and is also proportional to the elemental abundance. From Eq. (1), for any spectroscopic line, the loci of the following curves

$E M\left(T_{\mathrm{e}}\right)=4 \pi(1 \mathrm{AU})^{2} \frac{F}{G\left(T_{\mathrm{e}}, N_{\mathrm{e}}\right)}$

indicate the upper limit to the emission measure distribution. If the plasma is perfectly isothermal, all curves should intersect at a common point with coordinates at the temperature and emission measure of the isothermal plasma. The overlap between all curves also indicates whether the adopted relative abundances are correct. However, observational uncertainties need to be considered, and so the isothermal diagnostic curves would define a small region on the $E M$ vs. $T_{\mathrm{e}}$ plot, rather than one point. In the $E M$ loci analysis we used the measured fluxes of the lines indicated in Table 1. To calculate the $G\left(T_{\mathrm{e}}, N_{\mathrm{e}}\right)$ functions, we employed the CHIANTI routine gofnt which assumes a definition of the volume emission measure as $\int N_{\mathrm{e}} N_{\mathrm{H}} \mathrm{d} V$ and a value of 0.83 for $N_{\mathrm{H}} / N_{\mathrm{e}}$ (the number density of neutral hydrogen atoms relative to the number density of the electrons in the emitting plasma). Therefore, contribution functions calculated with this routine had to be divided by a factor of 0.83 in order to obtain the $G\left(T_{\mathrm{e}}, N_{\mathrm{e}}\right)$ corresponding to our definition of the emission measure $\left(\int N_{\mathrm{e}}^{2} \mathrm{~d} V\right)$.

\subsection{Continuum emission measure loci analysis}

One can distinguish between RESIK lines and continuum, both of which may be used as tools for diagnostics which complement 
Table 1. Observed RESIK spectral features. The check-marked lines were used in the EM loci analysis (see Sect. 5) for the rise (R), peak (P) and decay (D) phases of the 2003 Feb. 22 flare.

\begin{tabular}{|c|c|c|c|c|c|c|c|}
\hline $\begin{array}{l}\text { RESIK } \\
\text { Cha. No. }\end{array}$ & Ion & $\mathrm{R}$ & $\mathrm{P}$ & $\mathrm{D}$ & $\begin{array}{c}\lambda_{\text {pred }}(\AA) \\
\text { (CHIANTI v. 5.2) } \\
\end{array}$ & Transition & $\begin{array}{c}\log T_{\max }^{*} \\
(\mathrm{~K})\end{array}$ \\
\hline \multicolumn{8}{|l|}{1} \\
\hline & Ar XVII & & & & 3.365 & $1 \mathrm{~s}^{2}{ }^{1} \mathrm{~S}_{0}-1 \mathrm{~s} 3 \mathrm{p}{ }^{1} \mathrm{P}_{1}$ & 7.2 \\
\hline & Ar XVI Sat. & & & & 3.428 & $1 s^{2} 2 p^{2} P_{3 / 2}-1 s 2 p\left({ }^{3} P\right) 3 p^{2} D_{5 / 2}$ & 7.1 \\
\hline & K XVIII & $\checkmark$ & $\checkmark$ & & 3.532 & $1 \mathrm{~s}^{2}{ }^{1} \mathrm{~S}_{0}-1 \mathrm{~s} 2 \mathrm{p}^{1} \mathrm{P}_{1}$ & 7.2 \\
\hline & K XVIII & $\checkmark$ & $\checkmark$ & & 3.571 & $1 \mathrm{~s}^{2}{ }^{1} \mathrm{~S}_{0}-1 \mathrm{~s} 2 \mathrm{~s}^{3} \mathrm{~S}_{1}$ & 7.2 \\
\hline & S XVI & & & & $3.696,3.696$ & $1 \mathrm{~s}^{2} \mathrm{~S}_{1 / 2}-5 \mathrm{p}^{2} \mathrm{P}_{3 / 2,1 / 2}$ & 7.2 \\
\hline & S XVI & & & & $3.784,3.785$ & $1 \mathrm{~s}^{2} \mathrm{~S}_{1 / 2}-4 \mathrm{p}^{2} \mathrm{P}_{3 / 2,1 / 2}$ & 7.2 \\
\hline & \multicolumn{7}{|c|}{ 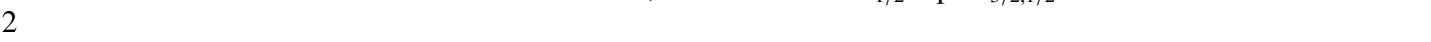 } \\
\hline & Ar XVII & $\checkmark$ & $\checkmark$ & $\checkmark$ & 3.949 & $1 \mathrm{~s}^{2}{ }^{1} \mathrm{~S}_{0}-1 \mathrm{~s} 2 \mathrm{p}{ }^{1} \mathrm{P}_{1}$ & 7.2 \\
\hline & & $\checkmark$ & $\checkmark$ & $\checkmark$ & 3.966 & $1 \mathrm{~s}^{2}{ }^{1} \mathrm{~S}_{0}-1 \mathrm{~s} 2 \mathrm{p}^{3} \mathrm{P}_{2}$ & 7.2 \\
\hline & & $\checkmark$ & $\checkmark$ & $\checkmark$ & 3.969 & $1 \mathrm{~s}^{2}{ }^{1} \mathrm{~S}_{0}-1 \mathrm{~s} 2 \mathrm{p}^{3} \mathrm{P}_{1}$ & 7.2 \\
\hline & S Xv & $\checkmark$ & $\checkmark$ & $\checkmark$ & 4.089 & $1 \mathrm{~s}^{2}{ }^{1} \mathrm{~S}_{0}-1 \mathrm{~s} 4 \mathrm{p}{ }^{1} \mathrm{P}_{1}$ & 7.2 \\
\hline \multicolumn{8}{|c|}{ 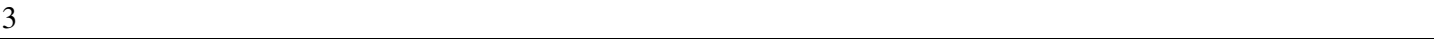 } \\
\hline & $\mathrm{S} \times \mathrm{V}$ & $\checkmark$ & $\checkmark$ & $\checkmark$ & 4.299 & $1 \mathrm{~s}^{2}{ }^{\mathrm{I}} \mathrm{S}_{0}-1 \mathrm{~s} 3 \mathrm{p}{ }^{1} \mathrm{P}_{1}$ & 7.2 \\
\hline & S XVI & $\checkmark$ & $\checkmark$ & $\checkmark$ & $4.727,4.733$ & $1 \mathrm{~s}^{2} \mathrm{~S}_{1 / 2}-2 \mathrm{p}{ }^{2} \mathrm{P}_{3 / 2,1 / 2}$ & 7.2 \\
\hline & Si XIV & & & & $4.831,4.831$ & $1 \mathrm{~s}^{2} \mathrm{~S}_{1 / 2}-5 \mathrm{p}{ }^{2} \mathrm{P}_{3 / 2,1 / 2}$ & 7.2 \\
\hline \multicolumn{8}{|c|}{ 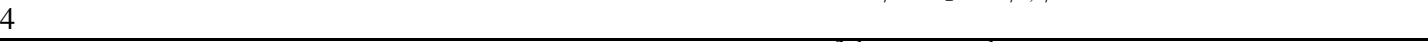 } \\
\hline & $\mathrm{S} \times \mathrm{V}$ & $\checkmark$ & $\checkmark$ & $\checkmark$ & 5.039 & $1 \mathrm{~s}^{2}{ }^{\mathrm{I}} \mathrm{S}_{0}-1 \mathrm{~s} 2 \mathrm{p}{ }^{1} \mathrm{P}_{1}$ & 7.1 \\
\hline & & $\checkmark$ & $\checkmark$ & $\checkmark$ & 5.102 & $1 \mathrm{~s}^{2}{ }^{1} \mathrm{~S}_{0}-1 \mathrm{~s} 2 \mathrm{p}^{3} \mathrm{~S}_{1}$ & 7.1 \\
\hline & Si XIV & $\checkmark$ & $\checkmark$ & $\checkmark$ & $5.217,5.218$ & $1 \mathrm{~s}^{2} \mathrm{~S}_{1 / 2}-3 \mathrm{p}{ }^{2} \mathrm{P}_{3 / 2,1 / 2}$ & 7.2 \\
\hline & Si XIII & & & & 5.286 & $1 \mathrm{~s}^{2}{ }^{1} \mathrm{~S}_{0}-1 \mathrm{~s} 5 \mathrm{p}{ }^{1} \mathrm{P}_{1}$ & 7.1 \\
\hline & Si XIII & $\checkmark$ & $\checkmark$ & $\checkmark$ & 5.405 & $1 \mathrm{~s}^{2}{ }^{1} \mathrm{~S}_{0}-1 \mathrm{~s} 4 \mathrm{p}{ }^{1} \mathrm{P}_{1}$ & 7.1 \\
\hline & & $\checkmark$ & $\checkmark$ & $\checkmark$ & 5.408 & $1 \mathrm{~s}^{2}{ }^{1} \mathrm{~S}_{0}-1 \mathrm{~s} 4 \mathrm{p}^{3} \mathrm{P}_{1}$ & 7.1 \\
\hline & Si XIII & $\checkmark$ & $\checkmark$ & $\checkmark$ & 5.681 & $1 \mathrm{~s}^{2}{ }^{1} \mathrm{~S}_{0}-1 \mathrm{~s} 3 \mathrm{p}^{1} \mathrm{P}_{1}$ & 7.1 \\
\hline & Si XII Sat. & & & $\checkmark$ & 5.816 & $1 \mathrm{~s}^{2} 2 \mathrm{p}^{2} \mathrm{P}_{1 / 2,3 / 2}-1 \mathrm{~s} 2 \mathrm{p}\left({ }^{3} \mathrm{P}\right) 3 \mathrm{p}^{2} \mathrm{D}_{3 / 2,5 / 2}$ & 7.0 \\
\hline
\end{tabular}

*Temperature of the fractional ion population peak from Mazzotta et al. (1998).

each other. We introduce a new technique for deriving plasma emission measure and temperature, based on the continuum $E M$ contribution. The method is analogous to the line-based emission measure analysis, with several segments of continuum being considered. The continuum contribution functions have a different shape and depend on the physical parameters of the flaring plasma in a different manner from the line contribution functions. Thus, by applying the EM loci analysis to the measured continuum fluxes in addition to the line intensities, we introduce a separate, independent means of characterising the flaring plasma. Furthermore, agreement between the line and continuum $E M$ loci indicates the correct elemental abundances relative to hydrogen.

The total continuum is defined as the sum of thermal freefree (bremsstrahlung), free-bound and two-photon continua. The continuum contributions are abundance-dependent with the twophoton continuum giving a negligible relative contribution (see e.g. White et al. 2005). The fluxes of the continuum segments used in the $E M$ loci analysis were measured by fitting the observed RESIK continuum in regions "free" of emission lines. We note that, strictly speaking, at the RESIK resolution, there are no regions entirely free of lines; however, we expect the line contribution in these regions to be relatively small.

\subsection{Differential emission measure analysis}

For a multi-thermal emitting plasma, the differential emission measure function $\operatorname{DEM}(T)$ is defined as

$\operatorname{DEM}(T)=N_{\mathrm{e}}^{2} \frac{\mathrm{d} V}{\mathrm{~d} T}$
The DEM may loosely be regarded as the amount of the emitting plasma within the instrument field of view (FOV) that has a temperature between $T$ and $T+\mathrm{d} T$ (for a physical interpretation of the DEM, see e.g. Sylwester et al. 1980). Assuming a constant abundance within the instrument FOV, the intensity of the observed flux can be rewritten as

$F=\frac{1}{4 \pi(1 \mathrm{AU})^{2}} \int G\left(T_{\mathrm{e}}, N_{\mathrm{e}}\right) D E M(T) \mathrm{d} T$.

To evaluate the $\operatorname{DEM}(T)$ distribution, we used an application of the "maximum entropy method", as described in Monsignori Fossi \& Landini (1991) and implemented by Del Zanna (1999). The DEM function is assumed to be a cubic spline with a limited number of selected "mesh" points, covering the temperature range where observational constraints are present. The rate of convergence is controlled by a lagrangian multiplier and the number of iterations. The fitting program allows one to search for the best solution for each single mesh point. We performed the DEM calculations using the fluxes of the lines indicated in Table 1 together with the measured fluxes of $0.2 \AA$ wide continuum bands in line-free continuum regions. Once an appropriate DEM was found, we calculated synthetic intensities in the RESIK wavelength range, in order to check if, overall, the observed spectra are well reproduced. The process was iterated several times, adjusting the mesh points and the element abundances to arrive at the best agreement between the observed and the synthetic spectra.

\subsection{Plasma diagnostics results}

Figure 7 shows the results of the line as well as continuum EM loci analyses described in Sects. 5.1 and 5.2 for the RESIK 
RISE
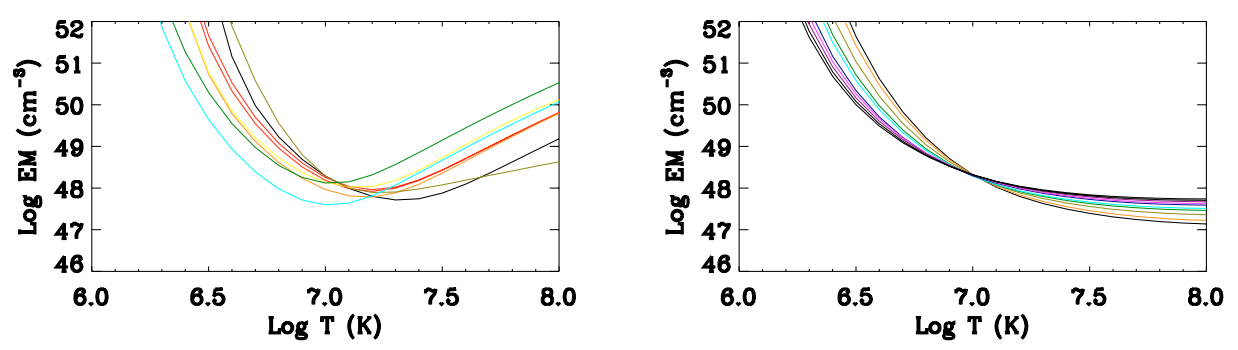

PEAK
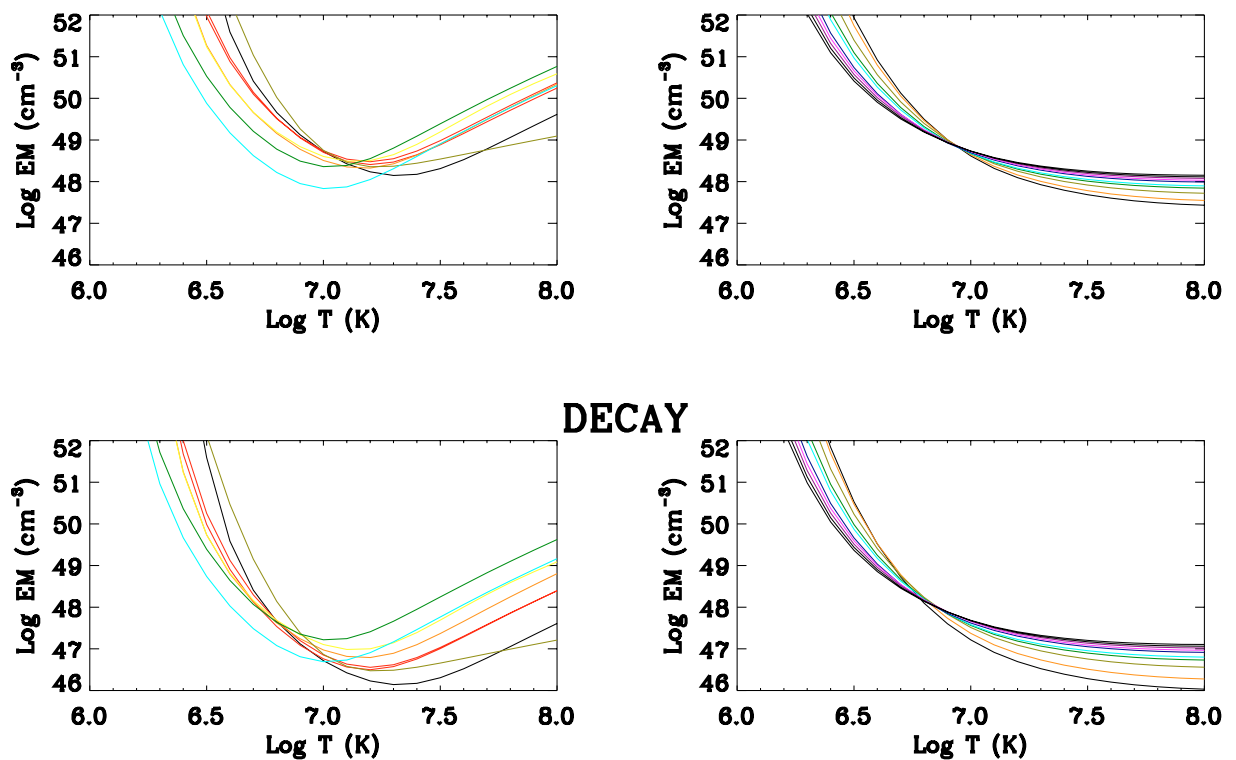

DECAY

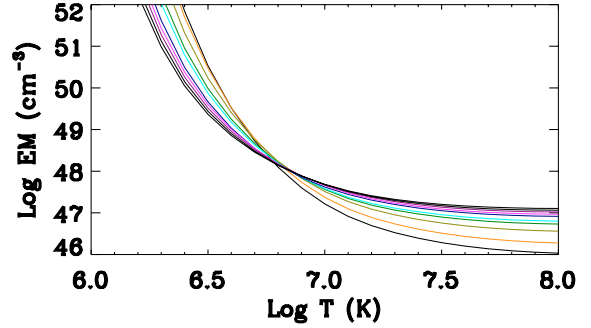

Fig. 7. Results from the RESIK line (left) and continuum (right) EM loci analyses. Agreement between the line and continuum EM loci required the use of photospheric abundances (Asplund et al. 2005). Averaged RESIK spectra were taken during the rise, peak and decay stages of the flare. The analysed RESIK lines are marked in Table 1. Each continuum curve corresponds to the observed flux within a $0.2 \AA$ wide band between 3.6 and $6.0 \AA$ A.

spectra taken during the 2003 Feb. 22 flare. In deriving the contribution functions for each observed emission lines and continuum band, we assumed the Mazzotta et al. (1998) ionisation equilibria. The line and continuum EM loci methods allowed a determination of the elemental abundances. Agreement between the line and continuum EM loci indicated photospheric abundances. The elemental abundances adopted in the present study are listed in Table 2. The EM derived with the loci method is $\sim 10^{48} \mathrm{~cm}^{-3}$ and does not fluctuate significantly throughout the evolution of the flare. Under an isothermal approximation, the temperature increases from approximately $10 \mathrm{MK}$ during flare rise to $12.6 \mathrm{MK}$ during peak flare emission, and then decays to about $7 \mathrm{MK}$. However, the intersection of the EM curves do not precisely constrain the temperature value and we find that the emitting flare plasma is more accurately described by a multithermal distribution with one dominant temperature component. The results of the DEM analysis outlined in Sect. 5.3. are plotted in Fig. 8. The derived DEM curves peak close to the temperatures indicated by the $E M$ loci analyses.

Assuming constant elemental abundances within the source volume, and using the DEM diagnostic method described in Sect. 5.3, we have derived, at the same time, abundances and the DEM distributions. The calculated DEM distributions were used to produce CHIANTI synthetic spectra which we compared with the observed RESIK spectra (Fig. 9). The adopted model

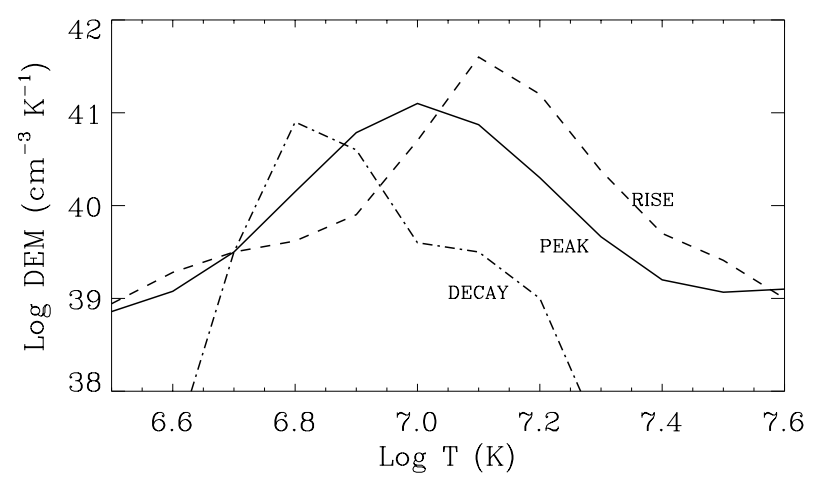

Fig. 8. DEM distributions calculated for the rise (dashed), peak (solid) and decay (dashes-dot) phases of the 2003 Feb. 22 flare from the observed RESIK lines and continua.

in obtaining the simulated intensities from the CHIANTI v. 5.2 database includes elemental abundances listed in Table 2, ionisation fractions from Mazzotta et al. (1998) and a constant density of $10^{10} \mathrm{~cm}^{-3}$. The synthetic line intensities were broadened with the empirically determined spectral resolution: $11.5,10.1,9.4$ and $15.7 \mathrm{~m} \AA$ (FWHM) in the four RESIK channels respectively.

The elemental abundances adopted in the present study are listed in Table 2. We note that in Asplund et al. (2005), 

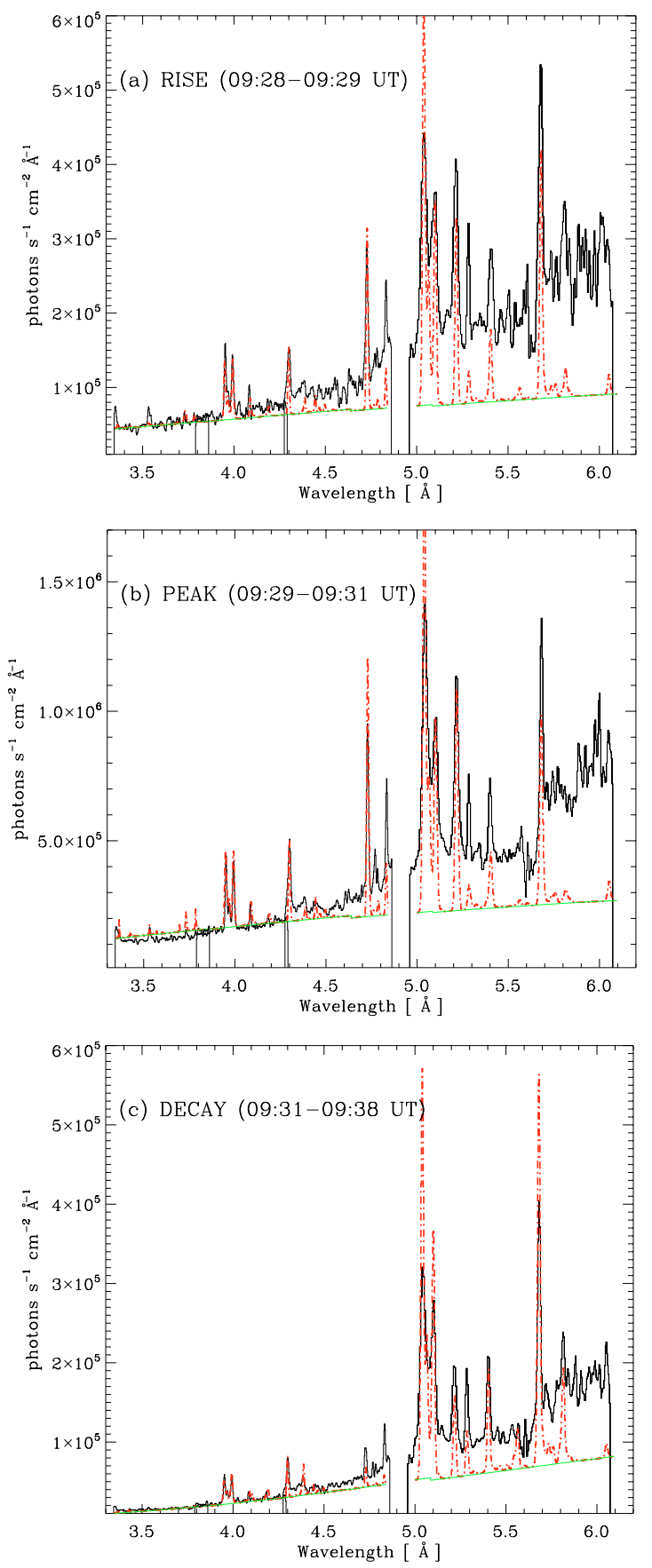

Fig. 9. RESIK observed spectrum (solid) with an overplotted simulated CHIANTI spectrum (dashed) during the flare rise a), peak b) and decay c). The CHIANTI spectrum was obtained from the derived differential emission measure curves using Mazzotta et al. (1998) ion fractions and the elemental abundances listed in Table 2. The synthetic lines where obtained using Gaussian profiles with the FWHM equal to the empirically determined RESIK instrumental resolution.

the abundances of some high-FIP elements have been considerably lowered, and that the Ar abundance was indirectly inferred. On the other hand, we provide a direct measurement of photospheric Ar abundances which are consistent with the value proposed by Asplund et al. (2005). The sulphur lines, however, indicate a considerable difference. We found it necessary to decrease the $S$ abundance of Asplund et al. (2005) by a factor of 2 (from $1.4 \times 10^{-5}$ to $7.9 \times 10^{-6}$ ) to bring the observed $\mathrm{S}$ line
Table 2. Elemental abundances adopted in this paper.

\begin{tabular}{lcc}
\hline \hline Element & $N(\mathrm{X})$ & $\log [N(\mathrm{X}) / N(\mathrm{H})] ; \log [N(\mathrm{H})]=12$ \\
\hline $\mathrm{Si}$ & $3.2 \times 10^{-5}$ & 7.51 \\
$\mathrm{~S}$ & $7.9 \times 10^{-6}$ & 6.90 \\
$\mathrm{Ar}$ & $1.5 \times 10^{-6}$ & 6.18 \\
$\mathrm{~K}$ & $1.2 \times 10^{-7}$ & 5.08 \\
\hline
\end{tabular}

intensities into agreement with the CHIANTI synthetic spectra. The S XV lines in RESIK channel 4 near $5 \AA$ (in particular, the $1 \mathrm{~s}^{2}{ }^{1} \mathrm{~S}_{0}-1 \mathrm{~s} 2 \mathrm{p}{ }^{1} \mathrm{P}_{1}$ line at $5.04 \AA$ ) are overestimated in all three flare stages, even using the depleted sulphur abundance.

Overall, the observed RESIK intensities are well represented by the synthetic spectra. However, the agreement in continua appears to decrease with wavelength, being worst in RESIK channel 4 (5.0-6.1 $\AA$ ). There are also features in the observed RESIK spectra which are very weak, particularly during the rise and peak phases of the flare. Some of these line features may be identified as e.g. Si XII $n=3$ and 4 dielectronic satellite lines at 5.82 and $5.56 \AA$ (Phillips et al. 2006a).

\section{Discussion and conclusions}

In this paper we carried out a benchmark analysis for the CHIANTI atomic database using observations from the RESIK instrument in order to check the validity of the CHIANTI data in the 3.4-6.1 $\AA$ range. An additional result of this work is a comparison between CHIANTI v. 5.2 and MEKAL which are found to be generally consistent in the RESIK range, noting, however, that the atomic data for the Si XII and Si XIII ions contained in CHIANTI are more complete.

The study has also allowed for the determination of the physical properties of the emitting plasma using three independent diagnostic techniques: EM loci analyses of RESIK lines and continuum and a DEM analysis. The line EM loci technique has been previously used in the EUV range, where continuum is small. Prior to applying this method to X-ray spectra the continua must be subtracted. The $E M$ contributions from the $\mathrm{X}$-ray continua can be used to complement the results from the line $E M$ loci as well as the DEM analyses.

The DEM analysis on the 2003 February 22 RESIK flare describes the multi-thermal nature of the emitting plasma, confirming the presence of a dominant temperature component, peaking close to the temperatures at which the RESIK line and continuum EM curves intersect. There is a very good agreement between the theoretical and observed continuum emission at the RESIK wavelengths of the first three channels. The higher observed continuum in channel 4 is probably due to fluorescence contamination still unaccounted for (Sylwester et al. 2005). However, there are also dielectronic satellites (e.g. Si XII $n=3$ and 4 at 5.82 and $5.56 \AA$ ) in this channel, which are not reproduced well except during the flare decay. These features are sensitive to temperatures as low as $3 \mathrm{MK}$ for which the derived DEM may not be well constrained during the rise and peak of the flare. The signal-to-noise ratio for channel 4 during the time intervals corresponding to the flare rise and peak did not allow us to distinguish these lines. We were only able to distinguish and include in our DEM analysis the Si XII satellite line at $5.82 \AA$ measured during flare decay (as shown in Table 1). It is probable that by introducing a low temperature component in the DEM functions calculated for the rise and peak of the flare, we could account for this discrepancy. Furthermore, during these 
impulsive flare phases, both continuum and dielectronic satellites may be subject to non-thermal effects (Dzif̌cáková \& Kulinová 2006).

The literature on elemental abundances in solar flare plasmas is vast. Somewhat surprisingly, the Si and Ar lines in the present study are very well represented with the use of the latest photospheric abundances (Asplund et al. 2005). On the other hand, the $\mathrm{S}$ abundance was found lower than the photospheric value by a factor of $\sim 2$. We note that this value $\left(7.9 \times 10^{-6}\right)$ is in excellent agreement with the one derived by Fludra \& Schmeltz (1999) using X-ray spectra from the Bragg Crystal Spectrometer (BCS) on Yohkoh, and close to the value previously found by these authors for the 1980 August 25 SMM flare $\left(9.7 \times 10^{-6}\right.$; Fludra \& Schmeltz 1995). Similarly to our study, Fludra \& Schmeltz (1995) used a DEM technique to obtain the abundances relative to hydrogen using $S M M / B C S$ spectra of lines and continua. Nevertheless, the depleted value of $S$ abundance did not satisfy the agreement between the predicted and observed S XV line at $5.04 \AA$ in any of the three flare phases, with the predicted line consistently underestimated. It is possible that, because the line is located near the low-wavelength edge of the RESIK channel, some edge effects may account for this disagreement.

The DEM analysis was carried out to characterise the multithermal composition, attempting to reproduce the majority of observed lines, but it is possible that there are also non-thermal effects which were not accounted for. The flare plasma most likely departs from being isothermal in the rise and peak stages of the flare.

Finally, our cross-calibration analysis revealed good agreement (particularly at flare peak) between recent well-calibrated RESIK spectra, RHESSI and GOES, confirming RESIK's precision (within $20 \%$ uncertainties) in calibration. This result confirms similar cross-calibrations by Sylwester et al. (2005) and Dennis et al. (2005) using preliminary RESIK spectra. On the other hand, discrepancies in temperatures are less pronounced in the late flare decay stage; this may be due to the non-isothermal nature of flare plasma in the impulsive flare stages and earlydecay stages. RHESSI is presumably observing a higher temperature component of the plasma.

RESIK spectral observations allow for an evaluation of any atomic database. The overall consistency between the CHIANTI v.5.2 simulated spectra and RESIK spectra is very satisfactory.

Acknowledgements. We would like to thank Dr. K. P. Dere for comments and suggestions which improved the quality of the manuscript. RESIK is a collaborative project between the Space Research Center (Poland), the Naval Research Laboratory (USA), the Mullard Space Science Laboratory (UK) and the Rutherford Appleton Laboratory (UK). The CHIANTI atomic database and code is an international project involving the Naval Research Observatory and George Mason University (USA), the Rutherford Appleton
Laboratory, the Mullard Space Science Laboratory and University of Cambridge (UK) and the University of Florence (Italy). C.C. is grateful for scholarship support received from the University of Cambridge Overseas Trust, an Isaac Newton Studentship from the Cambridge Institute of Astronomy and an Overseas Research Student Award. G.D.Z. thanks PPARC for its support and DAMTP, University of Cambridge, for its hospitality. H.E.M. acknowledges the support of PPARC. B.S. and J.S. acknowledge support from the Polish Ministry of Science grant 1.P03D.017.29.

\section{References}

Acton, L. W., Finch, M. L., Gilbreth, C. W., et al. 1980, Sol. Phys., 65, 53 Arnaud, M., \& Rothenflug, R. 1985, A\&AS, 60, 425

Asplund, M., Grevesse, N., \& Sauval, A. J. 2005, ASPC, 336, 25

Delaboudinière, J.-P., Artzner, G. E., Brunaud, J., et al. 1995, Sol. Phys., 162, 291

Del Zanna, G. 1999, Ph.D. Thesis, Univ. of Central Lancashire

Del Zanna, G. 2002, in Proc. of workshop on High resolution X-ray spectroscopy with XMM-Newton and Chandra, MSSL, 24-25 October 2002, ed. G. Branduardi-Raymont, 12

Del Zanna, G. 2006a, A\&A, 447, 761

Del Zanna, G. 2006b, A\&A, 459, 307

Del Zanna, G., Landini, M., \& Mason, H. E. 2002, A\&A, 385, 968

Del Zanna, G., Chidichimo, M. C., \& Mason, H. E. 2005, A\&A, 432, 1137

Dennis, B. R., Phillips, K. J. H., Sylwester, J., et al. 2005, Adv. Space Res., 35, 1723

Dere, K. P., Landi, E., Mason, H. E., Monsignori Fossi, B. C., \& Young, P. R. 1997, A\&AS, 125, 149

Dzifčáková, E., \& Kulinová, A., in Proc. of SoHO 17, ESA SP-617

Fludra, A., \& Schmelz, J. T. 1995, ApJ, 447, 936

Fludra, A., \& Schmelz, J. T. 1999, A\&A, 348, 286

Handy, B. N., Acton, L. W., Kankelborg, C. C., et al. 1999, Sol. Phys., 187, 229

Jordan, C., Ayres, T. R., Brown, A., Linsky, J. L., \& Simon, T. 1987, MNRAS, 225, 903

Landi, E., \& Phillips, K. J. H. 2005, ApJ, 160, 286

Landi, E., Del Zanna, G., Young, P. R., Dere, K. P., Mason, H. E., \& Landini, M. 2006, ApJS, 162, 261

Lin, R. P., Dennis, B. R., Hurford, G. J., et al. 2002, Sol. Phys., 210, 3

Mazzotta, P., Mazzitelli, G., Colafrancesco, S., \& Vittorio, N. 1998, A\&AS, 133, 403

Mewe, R., Schrijver, J., \& Sylwester, J. 1980, A\&A, 87, 55

Mewe, R., Gronenschild, E. H. B. M., \& van den Oord, G. H. J. 1985, A\&AS, 62, 197

Mewe, R., Kaastra, J. S., \& Liedahl, D. A. 1995, Legacy, 6, 16

Meyer, J. P. 1985, ApJSS, 57, 173

Monsignori Fossi, B. C., \& Landini, M. 1991, Adv. Space Res., 11, 281

Phillips, K. J. H. 2004, ApJ, 605, 921

Phillips, K. J. H., \& Landi, E. 2003, ApJ, 589, L113

Phillips, K. J. H., Harra-Murnion, L. K., Mewe, R., \& Kaastra, J. S. 1999a, ASP Conf. Ser., 158

Phillips, K. J. H., Mewe, R., Harra-Murnion, et al. 1999b, A\&A, 138, 381

Phillips, K. J. H., Dubau, J., Sylwester, J., \& Sylwester, B. 2006a, ApJ, 638, 1154

Phillips, K. J. H., Chifor, C., \& Dennis, B. R. 2006b, ApJ, 647, 1480

Smith, D. M., Lin, R. P., Turin, P., et al. 2002, Sol. Phys., 210, 33

Sylwester, J. Schrijver, J., \& Mewe, R. 1980, Sol. Phys., 67, 285

Sylwester, J., Gaicki, I., Kordylewski, Z., et al. 2005, Sol. Phys., 226, 45

Sylwester, B., et al. 2006a, in Proc. of IAU Symp., 233, in press

Sylwester, J., et al. 2006b, Adv. Space Res., submitted

White, S. M., Thomas, R. J., \& Schwartz, R. A. 2005, Sol. Phys., 227, 231 